\title{
Response of soil properties to yak grazing intensity in a Kobresia parva-meadow on the Qinghai-Tibetan Plateau, China
}

\author{
Q.M. Dong ${ }^{1}$, X.Q. Zhao ${ }^{2 *}$, G.L. Wu ${ }^{3}$, J.J. Shi ${ }^{1}$, Y.L. Wang1, L. Sheng ${ }^{1}$ \\ ${ }^{1}$ Qinghai Academy of Animal and Veterinary Sciences, Key Laboratory of Alpine Grassland Ecosystem in the \\ Three River Head Waters Region Jointly Funded by Qinghai Province and Ministry of Education, Xining, 810016 \\ Qinghai, P. R. China. ${ }^{2}$ Northwest Plateau Institute of Biology, Chinese Academy of Sciences, Xining, 810003 \\ Qinghai, P. R. China. ${ }^{3}$ State Key Laboratory of Soil Erosion and Dryland Farming on the Loess Plateau, Institute \\ of Soil and Water Conservation of Chinese of Academy of Sciences and Ministry of Water Resources / Northwest \\ A\&F University, Yangling 712100 Shaanxi, P.R. China *Corresponding author: xqzhao@nwipb.cas.cn
}

\begin{abstract}
Grazing intensity is one of the most important factors influencing soil properties variations in rangeland ecosystem. This research aimed to study the features of soil properties under different grazing intensity in a Kobresia parva-meadow on the Qinghai-Tibetan Plateau, China. Results showed that soil organic matter (SOM), soil organic carbon (SOC), and total nitrogen (N) significantly decreased with an increase grazing intensity and total and available potassium $(\mathrm{K})$, and $\mathrm{C} / \mathrm{N}$ ratio exhibited a similar pattern. However, there were not significant differences between warm-season pasture (WSP) and cool-season pasture (CSP). In addition, results indicated that soil $\mathrm{P}$ was a limited factor, and $\mathrm{N}$ was sensitive to grazing intensity in Kobresia parva alpine meadow grazing ecosystem. Therefore, our study demonstrated that soil properties, such as soil carbon and nitrogen, generally decreased with the increasing of grazing intensity in studied Kobresia parva-meadow on the Qinghai-Tibetan Plateau.
\end{abstract}

Keywords: Grazing intensity, biomass, soil properties, Kobresia parva-meadow, three-river headwaters region. 


\section{Introduction}

Grazing is the key driver of rangeland ecosystem structure and function, but its roles can be varied considerably in alpine rangeland ecosystems of the Qinghai-Tibetan Plateau (Harris, 2010). Studies had reported that grazing may negatively affect belowground organic matter, total and available nitrogen, total and available phosphorus (P) (Wu et al., 2009) and soil carbon storage (Sun et al., 2011). Grazing directly reduces aboveground biomass and increases light availability for shorter species in the vegetation, but whether grazing results in a reduction of soil nutrient availability (N, P and $\mathrm{K}$ ) -thus indirectly reducing biomass- are unknown. Milchunas and Lauenroth (1993) found, however, there was no effect on soil organic matter (SOM) and suggested that total nutrient stocks may undermine nutrient availability.

Overgrazing and soil-degradation are closely associated with each other (Xie and Wittig, 2004). This vegetation degradation related by overgrazing exposed the soil surface directly to wind and water erosion, leading to a loss of topsoil fertile and its nutrients and plants' seeds. In recent years, it suffers from seriously rangeland degradation, which mainly caused by grazing disturbance (Harris, 2010). In the alpine meadow ecosystem of this region, frigid climate and harsh natural conditions lead to a short growing period (90 120 d) and lower forage yield of plants, which caused unbalanced of seasonal pasture (warm-season and cold-season pasture). Moreover, with the effects of increasing yak population, global warming, and natural disasters, over half of rangeland has degraded at different level in this ecosystem. However, specific causes of degradation are still an active area of investigation (Klein et al., 2004; Harris, 2010). Livestock grazing is the dominant form of land use in alpine area of the Qinghai-Tibetan Plateau and Yak (Bos grunniens) is the typical grazing animal, playing a crucial role in the alpine meadow ecosystem and animal husbandry economy of this region. However, few studies have documented the effects of grazing intensity on the soil properties in the Kobresia parva-meadow on the Qinghai-Tibetan Plateau (Dong et al., 2005; Wu et al., 2009, 2010). In this study, we examined soil properties responses to grazing intensity in alpine rangeland ecosystem of the Qinghai-Tibetan Plateau. This work aimed to clarify the effects of grazing intensity on soil properties and to help guide grazing management. We specifically asked how grazing intensity influence soil properties in alpine rangeland ecosystems.

\section{Materials and Methods}

\subsection{Study site}

The experiment was carried out in Wosai Township of Dari County, Guoluo Tibetan Autonomous Prefecture of Qinghai Province, which is located at the southwest of the Qinghai-Tibetan Plateau $\left(99^{\circ} 47^{\prime} 38^{\prime \prime} \mathrm{N}\right.$, $\left.33^{\circ} 37^{\prime} 21^{\prime \prime} \mathrm{E}\right)$, with an average elevation of $4000 \mathrm{~m}$. The landscape is characterized by large mountain ranges with steep valleys and gorges interspersed with relatively level and wide inter-mountain rangeland basins. It has a continental monsoon-type climate, with severe and long winters, and short and cool summers. The average air temperature is $-1.3{ }^{\circ} \mathrm{C}$ with extremes of a maximum $24.6{ }^{\circ} \mathrm{C}$ and a minimum $-34.5{ }^{\circ} \mathrm{C}$. Average annual precipitation is about $590 \mathrm{~mm}, 80 \%$ of which falls in the short growing season from May to September; there is no absolute frost-free period. The annual average sunlight is 2331 hours. The main rangeland species are Kobresia parva, Kobresia humilis, Elymus nutans, Potentilla anserina, and Poa alpigena in moderately deteriorated state. The livestock 
assemblage in the Yangtze and Yellow rivers headwaters region includes yaks, Tibetan sheep, and horses. The local population is entirely Tibetan, and over $90 \%$ of the local inhabitants are pastoral.

\subsection{Experiment design}

To study the effects of yak grazing intensity on soil properties in two-season pastures (warm-season and cool-season pastures which refers when these pastures are grazed) in the study sites, we applied different levels of grazing intensity inside and outside enclosures from 1998 to 2000. After a preliminary survey of the vegetation according to forage yield, intake of growing yaks (2.4 kg $100 \mathrm{~kg}^{-1}$ live weight), area of plots and grazing intensities were determined. Three grazing treatments and one control treatment were selected within the area and fenced for warm season pasture (referred to hereafter as WSP) from 1998, where the block area was 4.5 ha, 2.75 ha, 1.92 ha, respectively for grazing blocks and 1.0 ha for the control block. Four yaks grazed in each of the grazing blocks from June 1 to October 31. For the cool season pasture (referred to hereafter as CSP), the grazing block areas were 5.19 ha, $3.09 \mathrm{ha}$, and $2.21 \mathrm{ha}$, and the control plot was $1.0 \mathrm{~h} \mathrm{~m}^{2}$. As with the WSP blocks, there were four yaks grazed in each of grazing blocks from November 1 to May 30 of the next year Outside of both WSP and CSP blocks, an area of $100 \mathrm{~m}$ x $100 \mathrm{~m}$ was identified to provide "free grazing for yaks" according to the traditional grazing system. These two areas were not fenced. Three grazing treatments were (1) light (L) grazing with $30 \%$ relative utilization ( 0.89 heads $\left.\cdot \mathrm{ha}^{-1}\right)$; (2) moderate (M) grazing with $50 \%$ relative utilization $\left(1.45\right.$ heads $\left.\cdot \mathrm{ha}^{-1}\right)$; and (3) heavy $(\mathrm{H})$ grazing with $70 \%$ relative utilization ( 2.08 heads ha $^{-1}$ ). Control (CK) was $0 \%$ relative utilization, and in the native $(\mathrm{N})$ grazing intensity by local herdsman, and there were 2.50 heads $\cdot \mathrm{ha}^{-1}$ estimated by comparison with control. In CSP, relative utilization percentages were the same as those for WSP (Table 1).

Table 1. Descriptions of design of grazing animal numbers, study plot area, and grazing intensity gradients in this field experiment.

\begin{tabular}{lccccc}
\hline \multirow{2}{*}{ Treatment } & $\begin{array}{c}\text { No. of yaks per plot } \\
\text { (heads) }\end{array}$ & \multicolumn{2}{c}{$\begin{array}{c}\text { Area of per plot } \\
\text { (ha) }\end{array}$} & \multicolumn{2}{c}{$\begin{array}{c}\text { Grazing intensity } \\
\text { (heads ha } \mathbf{~ h}^{-\mathbf{1}}\end{array}$} \\
\cline { 2 - 6 } & & WSP & CSP & WSP & CSP \\
\hline Control (no grazing) (CK) & 0 & 1.00 & 1.00 & 0.00 & 0.00 \\
Light grazing (L) & 4 & 4.5 & 5.19 & 0.89 & 0.77 \\
Moderate grazing (M) & 4 & 2.75 & 3.09 & 1.45 & 1.29 \\
Heavy grazing (H) & 4 & 1.92 & 2.21 & 2.08 & 1.81 \\
Native grazing (N) & $2-3$ & 1.00 & 1.00 & 2.50 & 2.30 \\
\hline
\end{tabular}

*Grazing intensities were determined by aboveground biomass, areas of plots, and theoretical yak intake (2.4 kg 100 $\mathrm{kg}^{-1}$ live weight); warm-season pasture (WSP); control in cool-season pasture (CSP). 


\subsection{Soil sampling}

Soil sampling was only conducted in 199 and total 60 subquadrants per treatment were used. Diagonal transects $100 \mathrm{~m}$ long were arranged in a grid pattern in north-south and east-west directions in each grazing pasture. There were two diagonal transects in each grazing block, respectively. The ends of each transect were permanently marked with wooden stakes identified by metal tags. Five specific sites were determined along the intersection of the two diagonal transects at $50 \mathrm{~m}$ intervals, and each site had three quadrants that were $0.5 \mathrm{~m} \times 0.5 \mathrm{~m}$, each of which was divided into four subquadrants $0.25 \mathrm{~m}$ x $0.25 \mathrm{~m}$. Soil sampling were collected by using a soil auger $(0-5,5-10$, and $10-20 \mathrm{~cm}$ ) in late August of 1999, on $0.25 \mathrm{~m} \mathrm{x} 0.25 \mathrm{~m}$ at five sites along two diagonal transects in each grazing subquadrant after all plants' litters fell down the soil surface of the field. Soil hardness and $\mathrm{pH}$ of the upper soil surface at $0-5 \mathrm{~cm}$ were determined yearly in late August, as well as canopy cover at five sites located along two diagonal transects in each block on the WSP and CSP. Soil bulk density, pH value, and chemical parameters of the different soil layers $(0-5$, $5-10$, and $10-20 \mathrm{~cm}$ ) were analyzed by the methods of Agriculture Chemistry Council, Soil Science Society of China (1983). Soil hardness was measured directly from the profile wall using a TF-3 measuring device; $\mathrm{pH}$ value was measured in distilled water suspension with glass electrode (PHS-2). Others indexes were detected as following: SOM: wet oxidation; total N: Kjeldahl method; available N: extraction with $20 \% \mathrm{NaCl}$-solution, followed by a $\mathrm{ZnFeSO}_{4}$ reduction procedure; total $\mathrm{P}$ : photometer analysis after decomposition with concentrated $\mathrm{NaOH}$ solution; available P: photometer analysis after extraction with $\mathrm{NaHCO}_{3}$ solution; total $\mathrm{K}$ : flame photometer analysis following decomposition with $\mathrm{NaOH}$ solution; available $\mathrm{K}$ : flame photometer analysis after extraction with $\mathrm{NH}_{4}$-acetate.

\subsection{Statistical analyses}

The following methods were applied: (1) analysis of variance (ANOVA) for multivariate analysis and least significant difference (LSD) for multiple comparisons among soil hardness, and different nutrient contents of different soil layers, respectively, under different grazing intensity; and (2) regression analysis for the correlation between grazing intensity and concerned parameters and among different parameters under the same grazing intensity.

\section{Results}

Soil hardness in the upper layers was greatly increased in the WSP $(p<0.01)$ and presented a gradual increasing in the CSP $(p<0.05)$ with the increased grazing intensity (Figure 1). 


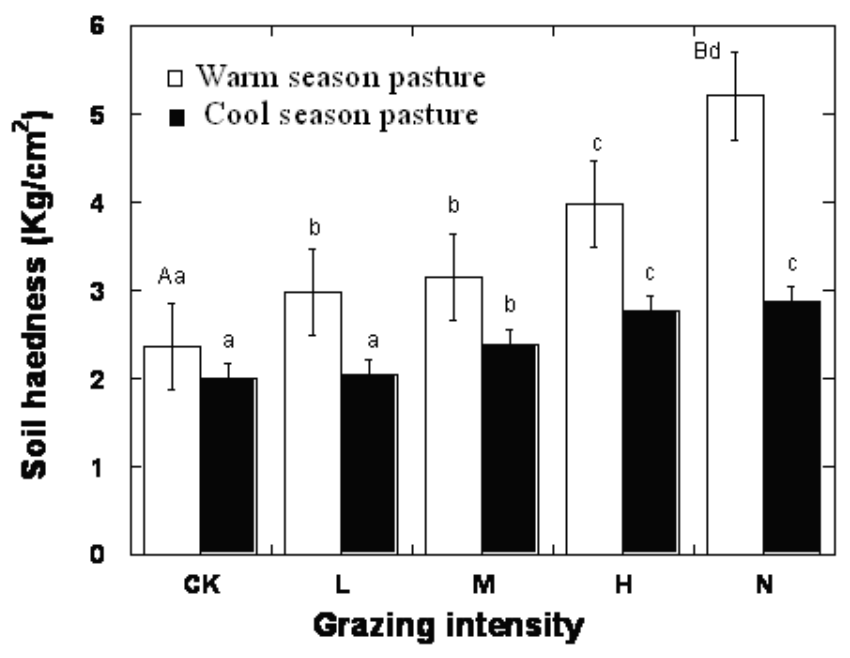

Figure 1. Changes of soil hardness under different grazing intensities in two-season pastures. Five grazing intensities were showed by Control (no grazing), CK; Light grazing, L; Moderate grazing, M; Heavy grazing, H; Native grazing, $\mathrm{N}$.

Soil bulk density showed a similar changing trend with soil hardness on the WSP and CSP (Table 2). Moreover, both WSP and CSP have the same trend with increased grazing activity on soil $\mathrm{pH}$ of upper layer to soil hardness (Figure 2).

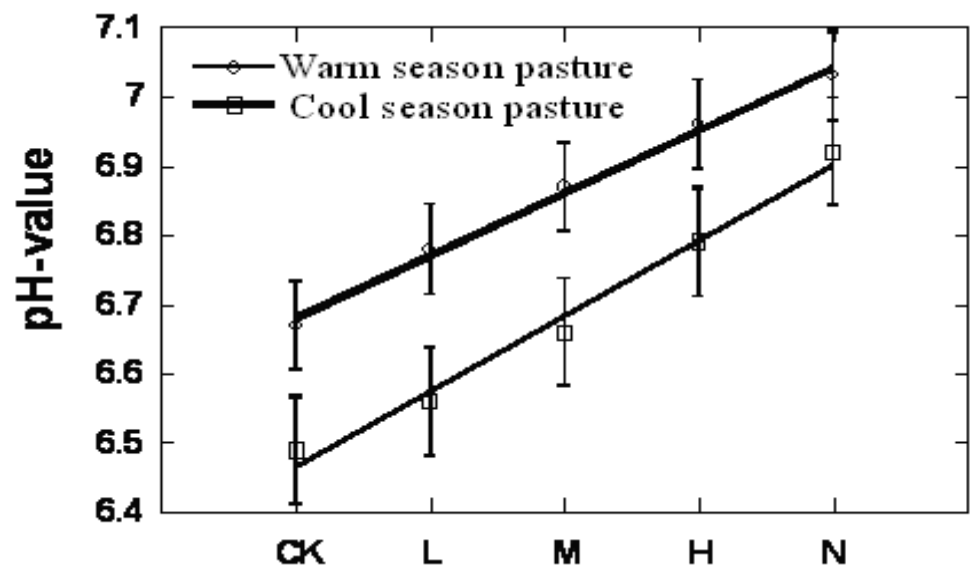

Figure 2. Changes of soil $\mathrm{pH}$ value under different grazing intensities in two-season pastures. Five grazing intensities were showed by Control (no grazing), CK; Light grazing, L; Moderate grazing, M; Heavy grazing, H; Native grazing, N. 
Table 2. Soil bulk density (Mean \pm standard error) under different grazing intensities (Control (no grazing) CK, Light grazing L, Moderate grazing M, Heavy grazing H, Native grazing N) in the warm-season pasture (WSP).

\begin{tabular}{lccccc}
\hline \multirow{2}{*}{ Depths(cm) } & \multicolumn{5}{c}{ Grazing intensity } \\
\cline { 2 - 6 } & $\mathbf{C K}$ & $\mathbf{L}$ & $\mathbf{M}$ & $\mathbf{H}$ & $\mathbf{N}$ \\
\hline $0-5$ & $1.45 \pm 0.19$ & $1.52 \pm 0.21$ & $1.60 \pm 0.21$ & $1.71 \pm 0.20$ & $1.99 \pm 0.07$ \\
$5-10$ & $1.69 \pm 0.23$ & $1.71 \pm .031$ & $1.87 \pm 0.34$ & $1.89 \pm 0.19$ & $2.28 \pm 0.09$ \\
$10-15$ & $1.71 \pm 0.21$ & $1.83 \pm 0.63$ & $1.95 \pm 0.21$ & $2.20 \pm 0.09$ & $2.36 \pm 0.13$ \\
\hline
\end{tabular}

SOM, SOC, and total soil nitrogen concentration at each depth $(0-5,5-10$, and $10-20 \mathrm{~cm})$ in WSP and CSP showed a declined trend with increasing of grazing intensity (Tables 3,4 ), and the multivariate analysis results showed that there were a significant differences among different grazing treatments and soil depths $(p<0.01$ for $0-5 \mathrm{~cm}$, and $p<0.05$ for 5-10 and $10-20 \mathrm{~cm}$ ) for SOM, SOC, and total soil nitrogen. Total $\mathrm{P}$ concentration of different soil depths exhibited a similar pattern of SOM, SOC, and total $\mathrm{N}$ in the WSP, and a significant difference was observed between different grazing treatments for the same soil depths $(p<0.05)$ (Table 3$)$. In the CSP, total $\mathrm{P}$ concentrations at the $0-5 \mathrm{~cm}$ depth decreased with increased grazing intensity $(p<0.05)$, and at the 5-10 and 10-20 depths were slightly higher in $\mathrm{M}$ than in $\mathrm{N}$, and lower than in CK. There was also a significant difference between the grazing levels at the same soil depths (Table 4) $(p<0.05)$. Total and available $\mathrm{K}$ concentrations at different depths showed a pattern similar to that of SOM, SOC, and total $\mathrm{N}$, but the differences were not significant under different grazing levels in the WSP and CSP $(p>0.05)$ (Tables 3, 4).

Table 3. Changes (Mean \pm standard error) in soil nutrients at different soil layers under stocking rates in the warm-season pasture. (Control (no grazing) CK, Light grazing L, Moderate grazing M, Heavy grazing H, Native grazing N).

\begin{tabular}{lcccc}
\hline \multirow{2}{*}{ Soil nutrient } & \multicolumn{4}{c}{ The depth of soil strata (cm) } \\
\cline { 2 - 5 } & Grazing intensity & $\mathbf{0 - 5} \mathbf{~ c m}$ & $\mathbf{5 - 1 0} \mathbf{~ c m}$ & $\mathbf{1 0 - 2 0} \mathbf{~ c m}$ \\
\hline Organic matter & $\mathrm{CK}$ & $156.81 \pm 49.72^{\mathrm{Aa}}$ & $97.73 \pm 30.07^{\mathrm{a}}$ & $72.92 \pm 25.03^{\mathrm{a}}$ \\
$\left(\mathrm{g} \mathrm{kg}^{-1}\right)$ & $\mathrm{L}$ & $130.73 \pm 32.74^{\mathrm{a}}$ & $90.93 \pm 21.98^{\mathrm{b}}$ & $59.82 \pm 8.73^{\mathrm{b}}$ \\
& $\mathrm{M}$ & $118.97 \pm 30.15^{\mathrm{b}}$ & $85.34 \pm 22.23^{\mathrm{b}}$ & $55.15 \pm 21.13^{\mathrm{b}}$ \\
& $\mathrm{H}$ & $103.43 \pm 39.05^{\mathrm{b}}$ & $78.12 \pm 26.33^{\mathrm{b}}$ & $45.83 \pm 21.24^{\mathrm{b}}$ \\
& $\mathrm{N}$ & $93.44 \pm 39.03^{\mathrm{Bb}}$ & $69.19 \pm 22.3^{\mathrm{b}}$ & $35.82 \pm 11.27^{\mathrm{b}}$ \\
Organic carbon & $\mathrm{CK}$ & $90.63 \pm 28.82^{\mathrm{Aa}}$ & $56.24 \pm 9.93^{\mathrm{a}}$ & $42.31 \pm 9.82^{\mathrm{a}}$ \\
$\left(\mathrm{g} \mathrm{kg}^{-1}\right)$ & $\mathrm{L}$ & $75.82 \pm 18.93^{\mathrm{b}}$ & $52.72 \pm 12.82^{\mathrm{a}}$ & $34.51 \pm 5.12^{\mathrm{b}}$ \\
& $\mathrm{M}$ & $68.92 \pm 17.52^{\mathrm{b}}$ & $49.51 \pm 1.12^{\mathrm{a}}$ & $31.93 \pm 9.95^{\mathrm{b}}$ \\
& $\mathrm{H}$ & $59.91 \pm 22.62^{\mathrm{b}}$ & $45.32 \pm 15.21^{\mathrm{b}}$ & $26.62 \pm 9.15^{\mathrm{c}}$ \\
& $\mathrm{N}$ & $53.91 \pm 20.67^{\mathrm{Bb}}$ & $35.31 \pm 11.41^{\mathrm{b}}$ & $21.62 \pm 4.11^{\mathrm{c}}$ \\
\hline
\end{tabular}


Continued...

\begin{tabular}{|c|c|c|c|c|}
\hline \multirow{2}{*}{ Soil nutrient } & \multicolumn{4}{|c|}{ The depth of soil strata $(\mathrm{cm})$} \\
\hline & Grazing intensity & $0-5 \mathrm{~cm}$ & $5-10 \mathrm{~cm}$ & $10-20 \mathrm{~cm}$ \\
\hline Total $\mathrm{N}$ & $\mathrm{CK}$ & $7.02 \pm 2.6^{\mathrm{a}}$ & $5.12 \pm 1.11^{\mathrm{a}}$ & $3.61 \pm 1.32^{\mathrm{a}}$ \\
\hline \multirow[t]{4}{*}{$\left(\mathrm{g} \mathrm{kg}^{-1}\right)$} & $\mathrm{L}$ & $6.21 \pm 2.12^{\mathrm{b}}$ & $4.43 \pm 1.92^{\mathrm{b}}$ & $3.23 \pm 1.22^{\mathrm{b}}$ \\
\hline & M & $5.93 \pm 1.61^{\mathrm{b}}$ & $3.92 \pm 1.75^{\mathrm{b}}$ & $3.05 \pm 1.21^{\mathrm{b}}$ \\
\hline & $\mathrm{H}$ & $5.41 \pm 2.0^{\mathrm{b}}$ & $4.22 \pm 1.18^{\mathrm{b}}$ & $2.62 \pm 1.86^{\mathrm{b}}$ \\
\hline & $\mathrm{N}$ & $5.21 \pm 1.09^{\mathrm{b}}$ & $3.27 \pm 1.08^{\mathrm{b}}$ & $2.22 \pm 1.16^{\mathrm{b}}$ \\
\hline Total P & CK & $2.52 \pm 1.01^{\mathrm{a}}$ & $2.41 \pm 1.12^{\mathrm{a}}$ & $2.23 \pm 0.81^{\mathrm{a}}$ \\
\hline \multirow[t]{4}{*}{$\left(\mathrm{g} \mathrm{kg}^{-1}\right)$} & $\mathrm{L}$ & $2.51 \pm 0.79^{\mathrm{a}}$ & $2.42 \pm 0.90^{\mathrm{a}}$ & $2.06 \pm 0.22^{\mathrm{a}}$ \\
\hline & M & $2.50 \pm 0.46^{\mathrm{a}}$ & $2.21 \pm .027^{\mathrm{a}}$ & $1.84 \pm 1.03^{\mathrm{a}}$ \\
\hline & $\mathrm{H}$ & $1.93 \pm 1.01^{\mathrm{b}}$ & $2.15 \pm 1.10^{\mathrm{a}}$ & $1.69 \pm 1.01^{\mathrm{b}}$ \\
\hline & $\mathrm{N}$ & $1.71 \pm 0.78^{\mathrm{b}}$ & $2.10 \pm 1.00^{\mathrm{b}}$ & $1.64 \pm 1.02^{\mathrm{b}}$ \\
\hline \multirow[t]{5}{*}{$\begin{array}{l}\text { Total K } \\
\left(\mathrm{g} \mathrm{kg}^{-1}\right)\end{array}$} & CK & $17.91 \pm 8.31^{\mathrm{a}}$ & $17.94 \pm 6.69^{\mathrm{a}}$ & $18.39 \pm 7.82^{\mathrm{a}}$ \\
\hline & $\mathrm{L}$ & $15.97 \pm 8.98^{\mathrm{a}}$ & $15.89 \pm 6.71^{\mathrm{a}}$ & $17.93 \pm 7.48^{\mathrm{a}}$ \\
\hline & M & $13.99 \pm 3.29^{\mathrm{a}}$ & $14.99 \pm 5.45^{\mathrm{a}}$ & $15.97 \pm 4.64^{\mathrm{a}}$ \\
\hline & $\mathrm{H}$ & $11.90 \pm 3.41^{\mathrm{a}}$ & $12.06 \pm 2.12^{\mathrm{a}}$ & $13.89 \pm 4.17^{\mathrm{a}}$ \\
\hline & $\mathrm{N}$ & $10.08 \pm 1.99^{\mathrm{a}}$ & $11.00 \pm 2.07^{\mathrm{a}}$ & $11.91 \pm 3.06^{\mathrm{a}}$ \\
\hline Available N & CK & $39.21 \pm 13.31^{\mathrm{Aa}}$ & $28.23 \pm 11.02^{\mathrm{a}}$ & $17.18 \pm 9.01^{\mathrm{a}}$ \\
\hline \multirow[t]{4}{*}{$\left(\mathrm{mg} \mathrm{g}^{-1}\right)$} & $\mathrm{L}$ & $38.83 \pm 15.32^{\mathrm{a}}$ & $23.52 \pm 10.34^{b}$ & $18.54 \pm 6.32^{\mathrm{a}}$ \\
\hline & M & $33.52 \pm 10.61^{\mathrm{a}}$ & $25.45 \pm 12.32^{\mathrm{a}}$ & $16.23 \pm 9.33^{\mathrm{a}}$ \\
\hline & $\mathrm{H}$ & $37.31 \pm 16.05^{\mathrm{a}}$ & $26.02 \pm 11.27^{\mathrm{a}}$ & $21.16 \pm 9.20^{\mathrm{b}}$ \\
\hline & $\mathrm{N}$ & $21.71 \pm 12.70^{\mathrm{Bb}}$ & $24.07 \pm 10.25^{\mathrm{b}}$ & $20.12 \pm 8.02^{b}$ \\
\hline Available P & $\mathrm{CK}$ & $0.24 \pm 0.09^{\mathrm{Aa}}$ & $0.20 \pm 0.10^{\mathrm{Aa}}$ & $0.22 \pm 0.11^{\mathrm{a}}$ \\
\hline \multirow[t]{4}{*}{$\left(\mathrm{mg} \mathrm{g}^{-1}\right)$} & $\mathrm{L}$ & $0.23 \pm 0.11^{\mathrm{a}}$ & $0.22 \pm 0.16^{\mathrm{a}}$ & $0.22 \pm 0.08^{\mathrm{a}}$ \\
\hline & M & $0.23 \pm 0.095^{\mathrm{a}}$ & $0.22 \pm 0.10^{\mathrm{a}}$ & $0.21 \pm 0.09^{\mathrm{a}}$ \\
\hline & $\mathrm{H}$ & $0.21 \pm 0.08^{\mathrm{a}}$ & $0.23 \pm 0.14^{\mathrm{a}}$ & $0.22 \pm 0.11^{\mathrm{a}}$ \\
\hline & $\mathrm{N}$ & $0.17 \pm 0.07^{\mathrm{Bb}}$ & $0.25 \pm 0.14^{\mathrm{Bb}}$ & $0.23 \pm 0.12^{\mathrm{b}}$ \\
\hline Available K & CK & $145.26 \pm 37.99^{\mathrm{a}}$ & $155.51 \pm 81.18^{\mathrm{a}}$ & $150.96 \pm 57.09^{\mathrm{a}}$ \\
\hline \multirow[t]{4}{*}{$\left(\mathrm{mg} \mathrm{g}^{-1}\right)$} & $\mathrm{L}$ & $139.05 \pm 22.09^{\mathrm{a}}$ & $159.61 \pm 79.99^{\mathrm{a}}$ & $149.99 \pm 35.08^{\mathrm{a}}$ \\
\hline & M & $135.89 \pm 42.28^{\mathrm{a}}$ & $147.29 \pm 60.44^{\mathrm{a}}$ & $140.59 \pm 60.18^{\mathrm{a}}$ \\
\hline & $\mathrm{H}$ & $124.88 \pm 59.61^{\mathrm{a}}$ & $135.98 \pm 36.99^{\mathrm{a}}$ & $139.15 \pm 35.12^{\mathrm{a}}$ \\
\hline & $\mathrm{N}$ & $114.29 \pm 39.19^{\mathrm{a}}$ & $124.98 \pm 35.09^{b}$ & $134.39 \pm 36.93^{\mathrm{a}}$ \\
\hline \multirow[t]{5}{*}{$\mathrm{C} / \mathrm{N}$} & $\mathrm{CK}$ & $12.91 \pm 3.91^{\mathrm{a}}$ & $11.74 \pm 2.92^{\mathrm{a}}$ & $11.08 \pm 2.82^{\mathrm{a}}$ \\
\hline & $\mathrm{L}$ & $12.16 \pm 2.62^{\mathrm{a}}$ & $12.04 \pm 3.04^{\mathrm{a}}$ & $10.82 \pm 2.51^{\mathrm{a}}$ \\
\hline & M & $12.7 \pm 2.71^{\mathrm{a}}$ & $12.56 \pm 2.67^{\mathrm{a}}$ & $10.43 \pm 3.22^{\mathrm{a}}$ \\
\hline & $\mathrm{H}$ & $11.11 \pm 1.92^{\mathrm{b}}$ & $10.93 \pm 1.96^{\mathrm{a}}$ & $10.26 \pm 2.11^{\mathrm{a}}$ \\
\hline & $\mathrm{N}$ & $10.67 \pm 3.89^{b}$ & $9.99 \pm 3.09^{\mathrm{a}}$ & $9.69 \pm 2.67^{\mathrm{a}}$ \\
\hline
\end{tabular}


Table 4. Changes (Mean \pm standard error) in soil nutrient at different soil strata under stocking rates in the cold-season pasture. (Control (no grazing) CK, Light grazing L, Moderate grazing M, Heavy grazing H, Native grazing N).

\begin{tabular}{|c|c|c|c|c|}
\hline \multirow{2}{*}{ Soil nutrient } & \multicolumn{4}{|c|}{ The depth of soil strata $(\mathrm{cm})$} \\
\hline & Grazing intensity & $0-5 \mathrm{~cm}$ & $5-10 \mathrm{~cm}$ & $10-20 \mathrm{~cm}$ \\
\hline Organic matter & $\mathrm{CK}$ & $173.12 \pm 78.09^{\mathrm{Aa}}$ & $117.98 \pm 38.99^{\mathrm{b}}$ & $80.36 \pm 29.12^{\mathrm{a}}$ \\
\hline \multirow[t]{4}{*}{$\left(\mathrm{g} \mathrm{kg}^{-1}\right)$} & $\mathrm{L}$ & $162.24 \pm 67.12^{\mathrm{Aa}}$ & $111.33 \pm 29.98^{b}$ & $75.03 \pm 23.46^{\mathrm{a}}$ \\
\hline & M & $149.77 \pm 56.12^{\mathrm{b}}$ & $90.23 \pm 33.01^{\mathrm{c}}$ & $60.16 \pm 30.10^{\mathrm{b}}$ \\
\hline & $\mathrm{H}$ & $143.96 \pm 41.67^{\mathrm{b}}$ & $86.12 \pm 29.61^{\mathrm{c}}$ & $54.80 \pm 19.99^{\mathrm{c}}$ \\
\hline & $\mathrm{N}$ & $123.61 \pm 51.09^{\mathrm{Bc}}$ & $70.12 \pm 19.51^{\mathrm{c}}$ & $44.83 \pm 14.09^{c}$ \\
\hline Organic carbon & $\mathrm{CK}$ & $111.03 \pm 23.67^{\mathrm{Aa}}$ & $68.92 \pm 22.21^{\mathrm{a}}$ & $42.91 \pm 21.01^{\mathrm{a}}$ \\
\hline \multirow[t]{4}{*}{$\left(\mathrm{g} \mathrm{kg}^{-1}\right)$} & $\mathrm{L}$ & $105.19 \pm 33.17^{\mathrm{a}}$ & $60.79 \pm 29.71^{\mathrm{a}}$ & $37.97 \pm 13.92^{\mathrm{a}}$ \\
\hline & M & $90.99 \pm 35.87^{\mathrm{b}}$ & $46.09 \pm 15.20^{\mathrm{b}}$ & $37.15 \pm 17.89^{\mathrm{a}}$ \\
\hline & $\mathrm{H}$ & $86.98 \pm 33.71^{\mathrm{b}}$ & $42.23 \pm 14.10^{\mathrm{b}}$ & $30.49 \pm 13.71^{\mathrm{b}}$ \\
\hline & $\mathrm{N}$ & $66.98 \pm 30.71^{\mathrm{Bb}}$ & $40.31 \pm 11.10^{\mathrm{b}}$ & $25.49 \pm 10.21^{\mathrm{b}}$ \\
\hline Total N & CK & $11.56 \pm 3.90^{\mathrm{Aa}}$ & $8.81 \pm 2.01^{\mathrm{b}}$ & $4.11 \pm 2.01^{\mathrm{c}}$ \\
\hline \multirow[t]{4}{*}{$\left(\mathrm{g} \mathrm{kg}^{-1}\right)$} & $\mathrm{L}$ & $10.22 \pm 2.91^{\mathrm{a}}$ & $7.98 \pm 3.12^{\mathrm{b}}$ & $3.67 \pm 1.97^{\mathrm{c}}$ \\
\hline & M & $8.47 \pm 2.07^{\mathrm{b}}$ & $6.33 \pm 2.09^{\mathrm{c}}$ & $3.33 \pm 1.52^{\mathrm{c}}$ \\
\hline & $\mathrm{H}$ & $8.03 \pm 1.98^{\mathrm{b}}$ & $5.99 \pm 2.51^{\mathrm{c}}$ & $3.38 \pm 1.17^{\mathrm{c}}$ \\
\hline & $\mathrm{N}$ & $7.34 \pm 1.88^{\mathrm{Bb}}$ & $4.09 \pm 1.57^{\mathrm{c}}$ & $2.68 \pm 1.32^{\mathrm{c}}$ \\
\hline Total P & $\mathrm{CK}$ & $2.73 \pm 1.01^{\mathrm{a}}$ & $2.23 \pm 1.00^{\mathrm{b}}$ & $2.01 \pm 0.99^{\mathrm{a}}$ \\
\hline \multirow[t]{4}{*}{$\left(\mathrm{g} \mathrm{kg}^{-1}\right)$} & $\mathrm{L}$ & $2.31 \pm 0.91^{\mathrm{a}}$ & $1.67 \pm 0.71^{\mathrm{b}}$ & $1.04 \pm 0.51^{\mathrm{b}}$ \\
\hline & M & $1.97 \pm 1.10^{\mathrm{b}}$ & $2.01 \pm 1.13^{\mathrm{b}}$ & $1.45 \pm 0.71^{\mathrm{b}}$ \\
\hline & $\mathrm{H}$ & $1.71 \pm 1.01^{\mathrm{b}}$ & $1.63 \pm 0.19^{\mathrm{b}}$ & $1.01 \pm 0.19^{\mathrm{b}}$ \\
\hline & $\mathrm{N}$ & $1.54 \pm 0.81^{\mathrm{b}}$ & $1.35 \pm 0.13^{\mathrm{c}}$ & $0.78 \pm 0.11 \mathrm{~B}^{\mathrm{b}}$ \\
\hline Total K & $\mathrm{CK}$ & $18.90 \pm 9.01^{\mathrm{a}}$ & $18.04 \pm 7.99^{\mathrm{a}}$ & $17.99 \pm 7.12^{\mathrm{a}}$ \\
\hline \multirow[t]{4}{*}{$\left(\mathrm{g} \mathrm{kg}^{-1}\right)$} & $\mathrm{L}$ & $17.70 \pm 8.98^{\mathrm{a}}$ & $17.09 \pm 8.01^{\mathrm{a}}$ & $16.90 \pm 7.08^{\mathrm{a}}$ \\
\hline & M & $12.09 \pm 4.89^{\mathrm{a}}$ & $14.98 \pm 5.99^{\mathrm{a}}$ & $13.07 \pm 4.92^{\mathrm{a}}$ \\
\hline & $\mathrm{H}$ & $10.43 \pm 5.01^{\mathrm{a}}$ & $10.76 \pm 4.08^{\mathrm{a}}$ & $11.89 \pm 5.67^{\mathrm{a}}$ \\
\hline & $\mathrm{N}$ & $9.98 \pm 2.09^{\mathrm{a}}$ & $9.80 \pm 3.67^{\mathrm{a}}$ & $10.01 \pm 3.76^{\mathrm{a}}$ \\
\hline Available N & $\mathrm{CK}$ & $31.91 \pm 16.7^{\mathrm{a}}$ & $23.92 \pm 10.72^{\mathrm{b}}$ & $17.03 \pm 7.32^{\mathrm{a}}$ \\
\hline \multirow[t]{4}{*}{$\left(\mathrm{mg} \mathrm{g}^{-1}\right)$} & $\mathrm{L}$ & $37.31 \pm 20.00^{\mathrm{a}}$ & $30.41 \pm 11.13^{\mathrm{a}}$ & $19.17 \pm 7.61^{\mathrm{a}}$ \\
\hline & M & $35.32 \pm 15.62^{\mathrm{a}}$ & $27.72 \pm 9.16^{\mathrm{a}}$ & $17.15 \pm 8.34^{\mathrm{a}}$ \\
\hline & $\mathrm{H}$ & $30.90 \pm 14.91^{\mathrm{a}}$ & $23.33 \pm 10.85^{\mathrm{b}}$ & $15.63 \pm 8.12^{\mathrm{a}}$ \\
\hline & $\mathrm{N}$ & $25.93 \pm 11.95^{\mathrm{b}}$ & $21.31 \pm 8.81^{\mathrm{b}}$ & $13.61 \pm 6.11^{\mathrm{b}}$ \\
\hline Available P & $\mathrm{CK}$ & $0.34 \pm 0.16^{\mathrm{a}}$ & $0.32 \pm 0.14^{\mathrm{a}}$ & $0.32 \pm 0.13^{\mathrm{a}}$ \\
\hline$\left(\mathrm{mg} \mathrm{g}^{-1}\right)$ & $\mathrm{L}$ & $0.33 \pm 0.11^{\mathrm{a}}$ & $0.30 \pm 0.16^{\mathrm{a}}$ & $0.32 \pm 0.11^{\mathrm{a}}$ \\
\hline
\end{tabular}


Continued...

\begin{tabular}{lcccc}
\hline \multirow{2}{*}{ Soil nutrient } & \multicolumn{4}{c}{ The depth of soil strata (cm) } \\
\cline { 2 - 5 } & Grazing intensity & $\mathbf{0 - 5} \mathbf{~ c m}$ & $\mathbf{5 - 1 0} \mathbf{~ c m}$ & $\mathbf{1 0 - 2 0} \mathbf{~ c m}$ \\
\hline $\mathrm{M}$ & $0.33 \pm 0.15^{\mathrm{a}}$ & $0.32 \pm 0.09^{\mathrm{a}}$ & $0.31 \pm 0.11^{\mathrm{a}}$ \\
& $\mathrm{H}$ & $0.31 \pm 0.17^{\mathrm{a}}$ & $0.33 \pm 0.14^{\mathrm{a}}$ & $0.32 \pm 0.09^{\mathrm{a}}$ \\
\hline Available K & $\mathrm{N}$ & $0.20 \pm 0.10^{\mathrm{b}}$ & $0.35 \pm 0.04^{\mathrm{b}}$ & $0.34 \pm 0.11^{\mathrm{b}}$ \\
$\left(\mathrm{mg} \mathrm{g}^{-1}\right)$ & $\mathrm{CK}$ & $165.06 \pm 34.99^{\mathrm{a}}$ & $175.56 \pm 88.98^{\mathrm{a}}$ & $155.56 \pm 67.89^{\mathrm{a}}$ \\
& $\mathrm{L}$ & $159.05 \pm 28.89^{\mathrm{a}}$ & $169.67 \pm 90.89^{\mathrm{a}}$ & $149.54 \pm 39.98^{\mathrm{a}}$ \\
& $\mathrm{M}$ & $155.89 \pm 72.98^{\mathrm{a}}$ & $157.90 \pm 70.34^{\mathrm{a}}$ & $150.09 \pm 80.78^{\mathrm{a}}$ \\
& $\mathrm{H}$ & $154.89 \pm 69.90^{\mathrm{a}}$ & $155.39 \pm 56.89^{\mathrm{a}}$ & $149.05 \pm 45.89^{\mathrm{a}}$ \\
\hline $\mathrm{C} / \mathrm{N}$ & $154.09 \pm 59.99^{\mathrm{a}}$ & $154.78 \pm 45.89^{\mathrm{a}}$ & $144.39 \pm 56.90^{\mathrm{a}}$ \\
\hline $\mathrm{N}$ & $13.91^{\mathrm{a}}$ & $12.82^{\mathrm{a}}$ & $11.08^{\mathrm{a}}$ \\
& $\mathrm{CK}$ & $13.67^{\mathrm{a}}$ & $11.98^{\mathrm{a}}$ & $10.87^{\mathrm{a}}$ \\
& $\mathrm{L}$ & $12.01^{\mathrm{b}}$ & $11.05^{\mathrm{b}}$ & $10.17^{\mathrm{b}}$ \\
$\mathrm{M}$ & $11.69^{\mathrm{b}}$ & $10.99^{\mathrm{b}}$ & $10.22^{\mathrm{b}}$ \\
& $\mathrm{H}$ & $10.78^{\mathrm{b}}$ & $9.09^{\mathrm{c}}$ & $9.99^{\mathrm{b}}$ \\
\hline
\end{tabular}

*as for each soil nutrient factor of same depth, different capital letters within columns are significantly different $(p<0.01)$; different small letters indicate significant difference $(p<0.05)$; the same small letters are no significant difference $(p>0.05)$.

Available $\mathrm{N}$ of all soil depths showed the highest in $\mathrm{L}$ treatment and the lowest in $\mathrm{N}$ in the CSP, and a significant difference was found among different grazing intensities in the CSP (Table 4) $(p<0.05)$. But in the WSP, available N concentration at the $0-5,5-10$, and $10-20 \mathrm{~cm}$ depths was the highest in $\mathrm{CK}$, and $\mathrm{HG}$ treatments, respectively. And the lowest in NG, LG, and MG treatments, respectively, which was significant different at all depths $(p$ $<0.01$ at $0-5 \mathrm{~cm}$ depth, $p<0.05$ at $5-10$ and $10-20$ cm depths) (Table 3).

Available $\mathrm{P}$ concentrate at the $0-5 \mathrm{~cm}$ depth exhibited a similar pattern to that of SOM, SOC, total N, total $\mathrm{K}$, and available $\mathrm{K}$ concentration, and a significant difference was found among different treatments in the CSP and WSP $(p<0.05)$ (Tables 3, 4). At the 5-10 $\mathrm{cm}$ depth, however, available $\mathrm{P}$ was the highest in NG treatment and the lowest in CK in the WSP, and the highest in NG and the lowest in LG treatment in the CSP, and a significant difference was found among different treatments in the WSP and CSP $(p<0.05)$. As for available $\mathrm{P}$ concentration in the $10-20 \mathrm{~cm}$ depths, it was the highest in NG treatment and the lowest in MG treatment, and there was a significant difference between different grazing treatments in the WSP and CSP $(p<0.05)$.

\section{Discussion}

Our results showed that grazing intensity had significantly negative effects on soil physical and nutrient properties. Our results suggested that soil hardness and $\mathrm{pH}$ in the upper layers of different grazing treatments in an alpine meadow ecosystem showed an increasing trend with increased grazing activity, but a significant difference for hardness was observed between grazing treatments in WSP and CSP. This was the same as soil bulk density but the differences were small and not significant, and this region was representative of non-saline soils. Furthermore, $\mathrm{Su}$ 
et al., (2005) had reported that grazed grasslands contained less organic $\mathrm{C}$ and more $\mathrm{N}$ than adjacent ungrazed rangeland. Wu et al., (2009) found that grazing showed significantly negative effects on soil organic matter, total nitrogen, available nitrogen, total phosphorus and available phosphorus than fencing rangeland in the Qinghai-Tibetan Plateau. Sun (2011) also reported that soil organic carbon storage during the plant growing seasons was also significantly reduced by grazing intensity.

What's more, it is said that high grazing intensity resulted in lower SOC and total $\mathrm{N}$ than low grazing intensity did. This may attribute to enhancing turnover of plant material and excreta and physical disruption of soil at high grazing pressure, which hastened the loss of soil C and N (Buxton et al., 1996; Wu et al., 2010). Continuous grazing resulted in less vegetation cover and litter accumulation, soil coarseness, and very low SOC and $\mathrm{N}_{\text {tot }}$ concentrations ( $\mathrm{Su}$ et al., 2005). The difference in $\mathrm{C} / \mathrm{N}$ ratio among grazing treatments at the same soil depths indicated that the impacts of continuous grazing on SOM exceed those of $\mathrm{N}_{\text {tot }}$ (Su et al., 2005). Moreover, the effect of grazing on denitrification was significant, and an increase in nitrogen loss through denitrification was found with increasing grazing intensity in the moist-cool winter and dry-warm summer. In our trial, $\mathrm{C} / \mathrm{N}$ ratio at different soil depths decreased with grazing intensity increased, but there was no significant difference and this conclusion was identical with the findings of Su et al., (2005).

Besides that, our founding released that total $\mathrm{P}$ decreased with the increase of stocking rates, and there was a significant linear regression relationship between total $\mathrm{P}$ content at each soil depth and stocking rates in the WSP. Our studies showed that grazing intensity significantly affected total and available $P$ content at the same soil depth. Furthermore, total and available K content decreased with the increased grazing activity, but the differences were limited and no significant changes, which was consistent with the findings of Xie and Wittig (2004) for the Stipa grandis and Stipa bungeana steppe in northern China, but quiet different with results of Kooijman and Smit (2001) found in acid dune grasslands of the Netherlands.

Additionally, vegetation cover decreases with grazing intensity increased in many studies of the alpine meadow of this area (Wu et al., 2009; 2010), and this further causes a decrease of microorganisms, mineralization velocity, and assimilation for plants and excretion for animals. The decreasing of soil organic matter, which is a major source of nutrient mineralization, may gradually lead to a smaller soil nutrient store and, thus to lower availability. In some grazing ecosystems research, however, grazing generally leads to a shift from competition for light to competition for nutrients, which allows more functionally different species to coexist (Berendse, 1985). Bezemer et al. (2006) had proposed that plant-soil feedback depends upon the plant functional group. Differences in plant functional groups can create different soil biotic and abiotic microhabitats by affecting soil organic matter, soil nutrient availability, and soil microbial communities (Casper and Castelli, 2007). Study found that GG species proportion were significant positively related to higher soil nutrient conditions (Wu et al., 2011). Meanwhile, grazing decreased the GG proportion and increased the FG proportion in alpine meadow communities, and there were lower soil nutrient contents in grazed meadows than in fenced meadows (Wu et al., 2009). Graminoid aboveground plant resources were transferred to the soil back when litter layers and roots decomposed (Bardgett and Wardle, 2003). These outcomes suggested that with the increasing of grazing intensity, recycling of aboveground plant and SOM, SOC, and total $\mathrm{N}$ to the soil was restricted. 


\section{Conclusions}

Grazing intensity changed soil properties by altering plant functional group compositions, their biomass loess and nutrient cycles in studied alpine rangeland ecosystem. Grazing intensity significant negatively impacted on soil physical and nutrient properties. Our results demonstrated that soil properties, such as soil carbon and nitrogen, generally decreased with the increasing of grazing intensity in studied Kobresia parva-meadow grazing ecosystem.

\section{Acknowledgements}

The authors thank Lang Baining, Zhou Li, and Liu Wei for their help in experiment design, and Li Youfu, Li Faji, Qia Jia, and Wang Changting for their help with field works, and thank Dr. Julia A. Klein and Paul Kretchmer and William D. Bowman for their assistance in editing this manuscript. This work was financially supported by National Key Sci-Tech Project (No. 2007BAC30B04) and Project of National Natural Science Foundation of China (30960074, 41030105, 30970519) the Qinghai Province Key SciTech Project.

\section{References}

Agriculture Chemistry Council, Soil Science Society of China. 1983. General analysis methods of soil agriculture chemistry. Science Press, Beijing. (In Chinese)

Bardgett, R.D., Wardle, D. 2003. Herbivore-mediated linkages between aboveground and belowground communities. Ecology 84, 2258-2268.

Berendse, F. 1985. The effect of grazing on outcome competition between plant species with different nutrient requirements. Okios 44, 35-39.

Bezemer, T.M., Lawson, C.S., Hedlund, K., Edwards, A.R., Brook, A.J., Igual, J.M., Mortimer, S.R., van der Putten, W.H. 2006. Plant species and functional group effects on abiotic and microbial soil properties and plant-soil feedback responses in two grasslands. Journal of Ecology 94, 893-904.

Buxton, D.R., Mertens, D.R., Fisher, D.S. 1996. Forage quality and ruminant utilization, In: Moser, L.E., Buxton, D.R., Casler, M.D. (Eds), Cool-season Forage Grasses. American Society of Agronomy, Madison, WI, 229-266.

Casper, B.B., Castelli, J.P. 2007. Evaluating plantsoil feedback together with competition in a serpentine grassland. Ecology Letters 10, 394-400.

Dong, Q.M., Ma, Y.S., Li, Q.Y., Zhao, X.Q., Wang, Q.J., Shi, J.J. 2005. Effects of Stocking rates for yak on community composition and plant diversity in Kobresia parva alpine meadow warm-season pasture. Acta Botany Boreal-Occident Sinica 25, 94-102. (In Chinese)

Harris, R.B. 2010. Rangeland degradation on the Qinghai-Tibetan plateau: A review of the evidence of its magnitude and causes. Journal of Arid Environments $74,1-12$.

Klein, J.A., Harte, J., Zhao, X.Q. 2004. Experimental warming causes large and rapid species loss, dampened by simulated grazing, on the Tibetan Plateau. Ecology Letters 7, 1170-1179.

Milchunas, D.G., Lauenroth, W.K. 1993. Quantitative effects of grazing on vegetation and soil over a global range of environments. Ecological Monographs 63, 327-366.

Su, Y.Z., Li, Y.L., Cui, J.Y., Zhao, W.Z. 2005. Infliences of continuous grazing and livestock exclusion on soil properties in a degraded sandy grassland, Inner Mongolia, northern China. Catena 59, 267-278.

Sun, D.S., Wesche, K., Chen, D.D., Zhang, S.H., Wu, G.L., Du, G.Z., Comerford, N.B. 2011. Grazing depresses soil carbon storage through changing plant biomass and composition in a Tibetan alpine meadow. Plant Soil Environment 57, 271-278.

Wu, G.L., Du, G.Z., Liu, Z.H., Thirgood, S. 2009. Effect of fencing and grazing on a Kobresia-dom- 
inated meadow in the Qinghai-Tibetan Plateau. Plant and Soil 319, 115-126.

Wu, G.L., Liu, Z.H., Zhang, L., Cheng, J.M., Hu, T.M. 2010. Long-term fencing improved soil properties and soil organic carbon storage in an alpine swamp meadow of western China. Plant and Soil 332, 331-337.

Wu, G.L., Li, W., Shi, Z.H, Shangguan, Z.P. (2011): Aboveground dominant functional group predicts belowground properties in an alpine grassland community of western China. Journal of Soils and Sediments 11, 1011-1019.

Xie, Y.Z., Wittig, R. 2004. The impact of grazing intensity on soil characteristics of Stipa grandis and Stipa bungeana steppe in northern China (autonomous region of Ningxia). Acta Oecologica 25, 197-204. 